\title{
Effects of Overlap Between Consecutive Words on Speeded Typing Inform About Representation of Serial Order Within Words
}

\author{
Fang Zhao ', Robert Gaschler', Teresa Travi', Birgit Imgrund ', Veronika Kossack', Eva \\ Röttger ${ }^{2}$, and Hilde Haider ${ }^{2}$
}

'Department of Psychology, FernUniversität in Hagen, Universitätsstraße 33, 58084, Hagen, Germany

2Department of Psychology, University of Cologne, Richard-Strauss-Straße 2, 50931, Cologne, Germany

ABSTRACT

Typing is an everyday activity that requires people to use the correct serial order of phonological and orthographic forms of words. The evidence until now shows that different forms of representation of serial order have mixed contributions to typing performance. It is not clear whether and how representational overlap between subsequent words impacts the speed of typing. In three experiments, we used speeded typing of six-letter words. Including conditions with secondary task load to counteract potential ceiling effects, we varied whether subsequent words had partial overlap with respect to a chaining representation (e.g., kirsch $\rightarrow$ schaum; same triplet in different position) or, in addition, overlapped with respect to a potential positional representation (e.g., berlin $\rightarrow$ dublin, same triplet in the same position). Differently from previous findings (e.g., Snyder \& Logan, 2014), Experiments 1 and 2 suggest that (a) chaining as well as positional coding are involved as representations of serial order and (b) partial overlap of representation of serial order leads to costs in typing speed. Experiment 3 demonstrated that full overlap speeds up typing. Across all experiments, the overlap effects were most revealed in the latency of the first keystroke, indicating the planning of motor programs. Taken together, the results suggest that even in highly practiced tasks such as typing, the activation of representations of serial order has side effects beyond the production of the current sequence.

representation of serial order, chaining, positional coding, typing,

dual-task effects

\section{INTRODUCTION}

Houghton and Hartley (1996) have used the example of typing words to disentangle different variants of representation of serial order in their seminal work. When typing the word word, people might, on the one hand, use associations between subsequent letters (e.g., $w \rightarrow o$, etc.). Empirical evidence for this type of representation (often referred to as chaining) has been shown as early as by Ebbinghaus (1885). On the other hand, people might represent words by associations between a letter and its position, for example, $w \rightarrow 1 ; o \rightarrow 2$ (e.g., Henson, 1998, for an overview on different variants of representation of serial order). The latter form of representation has been argued to cope with double letters (for instance, in letter; e.g., Kezilas, McKague, Kohnen, Badcock, \& Castles, 2017). By reviewing patterns in typing errors, Houghton and
Hartley (1996) underlined that (a) in typing, the letters of the entire word can be activated before the first key is pushed and that (b) the representation of serial order is needed first and foremost to solve the problem of serial order (Lashley, 1951): securing precise serial output in a parallel system with concurrently activated response options.

So far, evidence for the usage of different forms of representation of serial order in typing comes from two sources. On the one hand, analyses of typing errors have been used to support the relevance of representations other than chaining (e.g., Rumelhart \& Norman,

Corresponding author: Fang Zhao, Department of Psychology, University of Hagen, Universitätsstraße 33, D-58084 Hagen, Germany.

Email: fang.zhao@fernuni-hagen.de 
1982). For instance, transposition errors (e.g., tarp instead of trap; $a$ is pressed too early) have been used to argue for advanced activation based on associations of letter and position in the word (cf. Houghton \& Hartley, 1996). In the current paper, data were taken from continuous speeded typing. On the other hand, the representation of serial order in typing has been targeted for priming tasks (e.g., Snyder \& Logan, 2014). Preactivating the letters to be typed by showing an anagram did not speed up typing relative to a prime word with different letters. In tendency, people were even slowed down. When the prime featured a single letter in the position that would be due in the to-be-typed word, this was of no help either. Rather, participants were only speeded up by primes when these contained the same transitions of letters in the same position of the word.

Snyder and Logan (2014) investigated two interrelated questions. First, they scrutinized which type of representation of serial order is used to represent words in typing, suggesting that chaining plays a major role. Second, by testing priming effects, they documented that usage of these representations can be affected by information outside the current word. Given that typing can be considered a highly overlearned task, such a spillover is not trivial. One could have expected that the representation of the current word is robust enough. Typing is fast to an extent at which the overlap between the current word and the prime falls prey to a ceiling effect. However, unlike during continuous typing, participants experienced an alternation between primes and tobe-typed words. Thus, a stronger test for the impact of representations of serial order in a highly overlearned task such as typing would use typing without interruption.

Currently, it is not clear whether overlap between subsequent words in continuous speeded typing influences performance. Nor is it clear whether the impact of overlap would support or impede performance. Two routes of influence are conceivable when the last and the current word show partial overlap. The priming effects of Snyder and Logan (2014) suggest that leftover activation of the overlapping transitions might speed up typing. Alternatively, partial overlap could lead to costs. If the last and the current word overlap in parts, this might lead to sustaining or retrieving the prior word. This, in turn, could compete with typing the current word. Work on feature binding (Dreisbach \& Haider, 2009; Frings, Rothermund, \& Wentura, 2007; Hommel, 1998; Hydock, Patai, \& Sohn, 2013; Moeller, Pfister, Kunde, \& Frings, 2016; Stoet \& Hommel, 1999; Wiediger \& Fournier, 2008) shows that repetition versus alternation of features of a prior task affects performance in the current task. If enough of the features of the stimulus in the last trial repeat, people might retrieve the response that was due in the last trial but is not accurate in the current trial. Costs of partial overlap seem conceivable, given the evidence for word level effects of element activation. Perlman, Pothos, Edwards, and Tzelgov (2010) found priming effects of single element on the unit level, while Yamaguchi and Logan (2014) provided evidence for chunking effects. If people represent and access entire words in typing (rather than single letter transitions), partial overlap might lead to the activation of the wrong word and thus to costs in typing. Yamaguchi and Logan (2014) separately analyzed (a) word completion time as the overall typing speed, (b) latency of the first keystroke (and related it to higher-level processing such as encoding words and planning motor programs), and (c) interkeystroke interval as a measure of keystroke execution related to lower-level processing.

Taken together, the findings concerning chaining versus position coding suggest that the evidence is mixed for the contribution of different forms of representation underlying serial order in speeded typing. It is not clear whether the overlap of subsequent words leads to a benefit or to costs in performance. Across three experiments, we varied the overlap of words that were to be typed subsequently. In Experiments 1 and 2, the current six-letter word could either share a letter triplet (i.e., both contain lin or both contain $s c h$ ) with the previous word or have no such overlap. Same triplet in different position overlap would allow using chaining only (e.g., schaum, then kirsch). Same triplet in the same position overlap between subsequent words would allow using chaining codes and position representations (e.g., berlin, then dublin). In order to prevent the impact of overlap of representation of serial order between subsequent words being concealed by a ceiling effect, we included conditions with secondary task load. Given that Experiments 1 and 2 documented costs of partial overlap, we ran Experiment 3 to verify that full overlap (i.e., word repetition) indeed leads to a benefit in speed of typing.

\section{EXPERIMENT 1}

In Experiment 1, we varied the overlap between subsequent words in speeded typing. To avoid ceiling effects, participants had to conduct a (secondary) tone counting task. We used random sequences of sixletter words. All words contained either the triplet lin or sch in one of the four possible positions (start to end). This led to transitions with (a) no triplet overlap, (b) same triplet in different position, and (c) same triplet in the same position.

\section{Method}

\section{PARTICIPANTS}

Twenty-six German native speakers (13 females) participated in Experiment $1\left(M_{\text {age }}=41.1, S D=9.8\right)$ as volunteers for no extra reward. The mean age of participants was higher than in many laboratory studies in cognitive psychology, as students at FernUniversität in Hagen (state-run distance teaching university in Germany) are older and more heterogeneous in age than students at other universities. In the current study, we only required familiarity with the keyboard, but neither required nor assessed typing expertise. All participants had normal or corrected-to-normal vision acuity and normal hearing abilities.

\section{MATERIALS AND PROCEDURE}

We used continuous speeded typing of six-letter words as the primary task and tone counting as the secondary task. We used German words that either contained the triplet sch or lin. Both triplets are quite common in German. The triplet could be present either at the beginning of the word, start as Letter 2, as Letter 3, or be placed in the end 
of the word. For instance, we had schaum (foam), ascher (ashtray), dusche (shower), and kirsch (cherry, see Table 1). For each of the four possible triplet positions we chose three words from http://wortsuche. com/. This resulted in a list of 24 words ( 12 words for each of the two triplets; see Table 1).

The experiment was conducted on two identical laptops with German QWERTZ keyboard and 15.6 in. screens ( $1366 \times 768$ pixels). Tones were presented via headphones. Participants sat at a distance of $60 \mathrm{~cm}$ from the monitor. The words were all centrally presented in small letters $(0.7 \mathrm{~cm}$ height) in bold Courier New font by a Psychopy program (Peirce, 2007). There was one empty space between successive letters. Words were approximately $6.5 \mathrm{~cm}$ long. Only one word was presented each time on the display, in white against a black background. The to-be-typed letter was shown inside square brackets which moved rightward as soon as a keypress was registered (see Figure 1). The entire word was to be typed and participants could type in their own way (ten finger typing was not required). Participants were instructed to type as quickly and accurately as possible. When a word was finished, “ $>>$ ” appeared on the screen and participants were to press the space key to go to the next word. No error feedback was given.

Each of the 24 words was presented 8 times and participants continuously typed 192 words. Ninety six words were in block one and 96 words were in block two. The tasks were the same in both blocks: participants should type words and count the tones. The only differences were that the order of the words and the number of tones were different in both blocks. Subsequent words contained three types of overlap: (a) no overlap (e.g., dusche $\rightarrow$ linear), (b) same triplet in different position (which allowed using chaining only, e.g., ascher $\rightarrow$ kirsch), or (c) same triplet in the same position (which allowed using chaining codes and position representations, e.g., berlin $\rightarrow$ dublin). The random ordering of the words led to the no overlap condition occupying $55 \%$ of all trials, while the same triplet in different position condition had a $38 \%$ share and the same triplet in same position condition - the remaining 7\%. Rather than presenting prime-probe pairs (see, e.g., Snyder \& Logan, 2014), participants had to react to all words by typing them continuously. With the exception of the first word in the block, all the words served as primes and targets. As all words were presented randomly and never repeated, each word could be preceded by a word either containing the same triplet in the same or a different position in the word-or by a word not containing the same triplet. We coded overlap in an ad hoc manner in the continuous stream of typed words. The random ordering avoided leading participants to prepare for specific overlap conditions. By studying the effect of overlap between the previous word and the current word, we effectively compared how one and the same word is being typed depending on the overlap between the to-be-typed word and the preceding word. We therefore did not have to rely on comparisons between words, which might be influenced by word frequency effects.

For the secondary task, participants were required to count tones (played at $440 \mathrm{~Hz}$, lasting $500 \mathrm{~ms}$ ). Tones were placed randomly during typing such that one block contained 24 tones and the other block contained 25 tones (counterbalanced across participants). When typing a word, the tone onset could occur at any position in the word (but maximum one tone per word).

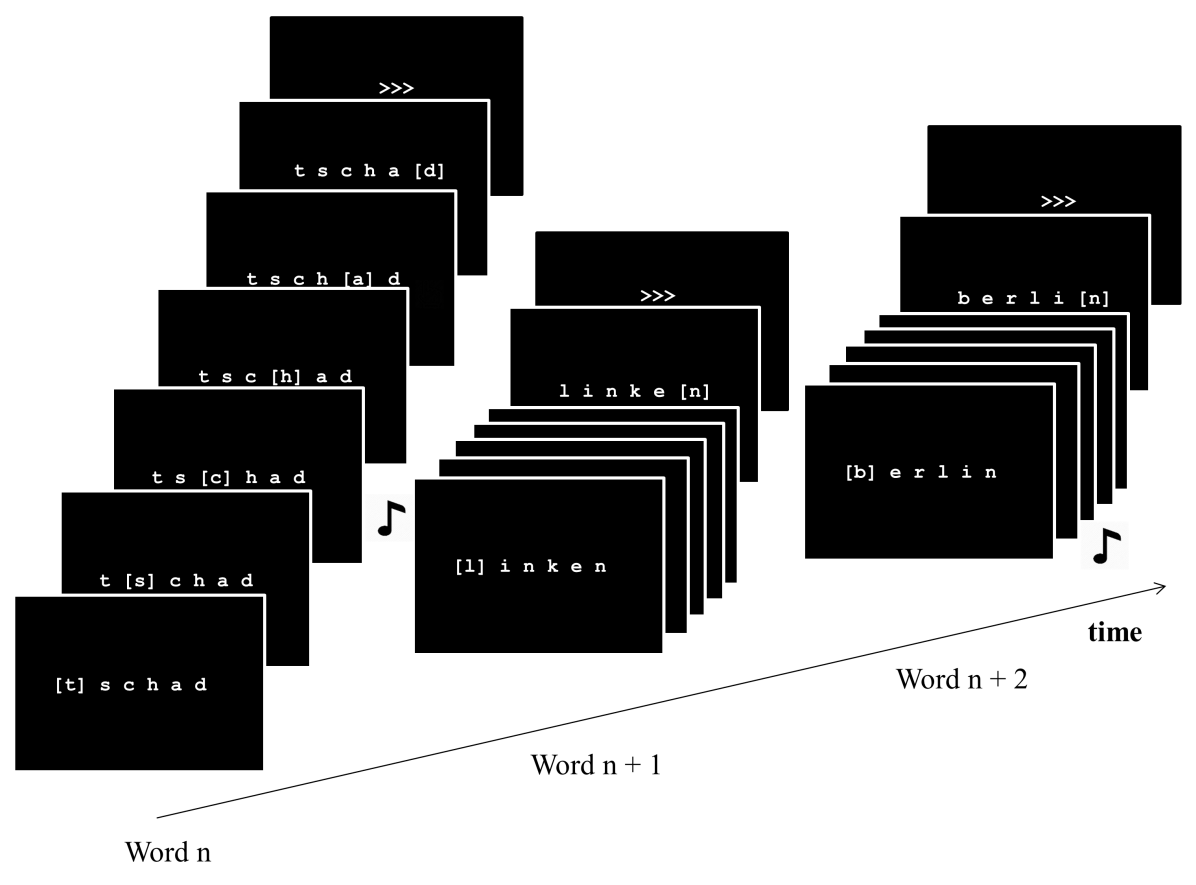

|FIGURE 1.

Task materials in the typing task (Task 1) and the tone counting task (marked by a music note as Task 2). The to-be-typed letter is marked by "[ ]". Only one word is presented each time on the display. Each word is separated by " $>>>$ ". As word $n$ was tschad and word $n+1$ was linken, the overlap condition is no overlap. As word $n+1$ was linken and word $n+2$ was berlin, overlap condition is same triplet in the different position. 


\begin{tabular}{|c|c|c|c|}
\hline \multicolumn{2}{|c|}{$\mathrm{SCH}$} & \multicolumn{2}{|c|}{ LIN } \\
\hline $\operatorname{sch}^{\star * *}$ & schaum & $\operatorname{lin}^{* * *}$ & linken \\
\hline & scherz & & lineal \\
\hline \multirow[t]{2}{*}{${ }^{*} \operatorname{sch}^{* *}$} & $\begin{array}{l}\text { schote } \\
\text { ascher }\end{array}$ & ${ }^{*} \operatorname{lin}^{* *}$ & $\begin{array}{l}\text { linear } \\
\text { clinch }\end{array}$ \\
\hline & ischia & & flinte \\
\hline \multirow[t]{2}{*}{${ }^{* *} \operatorname{sch}^{*}$} & $\begin{array}{l}\text { tschad } \\
\text { dusche }\end{array}$ & ${ }^{* *} \operatorname{lin}^{*}$ & $\begin{array}{l}\text { glinka } \\
\text { reling }\end{array}$ \\
\hline & gischt & & splint \\
\hline \multirow[t]{3}{*}{${ }^{* * *}$ sch } & $\begin{array}{l}\text { jascha } \\
\text { kirsch }\end{array}$ & ${ }_{* * *} \operatorname{lin}$ & $\begin{array}{l}\text { saline } \\
\text { dublin }\end{array}$ \\
\hline & barsch & & berlin \\
\hline & mensch & & myelin \\
\hline
\end{tabular}

\section{Results}

\section{SCREENING OF THE DATA}

The rate of words containing at least one error was $11.96 \%$ $(S D=7.92 \%)$. The mean difference between the correct number of tones and the reported number was $9.4 \%$. In tendency, people typing faster produced a higher proportion of words with errors, $r(26)=-.3, p=.135$. Less than one percent (i.e., $0.14 \%$ ) of the keystrokes were slower than $3 \mathrm{~s}$ and $5.12 \%$ were incorrect and excluded from analyses.

\section{WORD COMPLETION TIME}

Participants were faster to complete words with no overlap ( $M=2196 \mathrm{~ms}, S D=571 \mathrm{~ms})$ than words with the same triplet in different position $(M=2278 \mathrm{~ms}, S D=608 \mathrm{~ms})$, and slowest when the same triplet was repeated in the same position $(M=2361 \mathrm{~ms}$, $S D=639 \mathrm{~ms}), F(1.52,37.99)=17.95, p<.001, \eta_{\mathrm{p}}^{2}=.418$, for the repeated-measures analysis of variance (ANOVA; here and elsewhere we applied the Greenhouse-Geisser correction when appropriate). Two-tailed $t$-tests yielded the following results: $t(25)=4.51, p<.001$, $d=0.88^{1}$ for no overlap condition compared with same triplet in different position condition, $t(25)=5.21, p<.001, d=1.02$ for no overlap compared with same triplet in the same position condition, and $t(25)=2.7$, $p=.012, d=0.53$ for the difference between partial overlap at the same versus a different position. Next, we separately analyzed the latency of the first keystroke and the interkeystroke intervals (cf. Yamaguchi \& Logan, 2014) in order to determine whether overlap influenced latency to start typing a word and/or the execution of word typing.

\section{LATENCY OF THE FIRST KEYSTROKE}

Latency of the first keystroke was affected by word overlap, $F(1.28,32.04)=4.91, p=.026, \eta_{p}^{2}=.164$. As shown in Table 2, typing started later in words that contained the same triplet in the same position $(M=1089 \mathrm{~ms}, S D=309 \mathrm{~ms})$ than words with no overlap $(M=1045 \mathrm{~ms}, S D=249 \mathrm{~ms}, t[25]=2.65, p=.014, d=0.52)$. The latencies in case of the overlap of the triplet in different position $(M=1051 \mathrm{~ms}, S D=256 \mathrm{~ms})$ did not differ from no overlap $(p=.427)$, but were, in tendency, shorter than the overlap of the triplet and position, $t(25)=2, p=.056, d=0.39 .^{2}$

\section{INTERKEYSTROKE INTERVAL}

The interkeystroke interval was affected by overlap as well, $F(2,50)=11.91, p<.001, \eta_{\mathrm{p}}{ }^{2}=.323$. While no overlap $(M=222 \mathrm{~ms}$, $S D=71 \mathrm{~ms}$ ) was faster than the same triplet in different position condition $(M=237 \mathrm{~ms}, S D=78 \mathrm{~ms}, t[25]=4.66, p<.001, d=0.91)$ and the same triplet in the same position condition $(M=240 \mathrm{~ms}, S D=73 \mathrm{~ms}$, $t[25]=4.39, p<.001, d=0.86$ ), there was no difference between triplet overlap in the same versus a different position $(p=.476)^{3}$.

Taken together, this suggests that the extra delay of word completion in case a triplet was repeated in the same position originated from increased latency of the first keystroke. Thus, the overlap relevant for position coding seemed to play out in latency of the first keystroke, while the overlap relevant for chaining seemed to affect the interkeystroke intervals as well.

\section{Discussion}

The present experiment demonstrated an effect of partial overlap of subsequently typed words with respect to the representation of serial order in speeded continuous typing. In contrast to the priming paradigm used by Snyder and Logan (2014) and in line with unitization of sequence knowledge proposed by Hoffman et al. (2017), this overlap in continuous typing resulted in costs rather than a benefit in typing speed. When typing a word that shared a triplet with the preceding six-letter word, participants were slower. Furthermore, Snyder and Logan had argued that chaining is effective as a representation of serial order in typing. No effect of position coding could be detected in the priming task. However, we obtained evidence that position coding was used in addition to chaining as overlap matching both rather than only one of the forms of representations of serial order led to stronger slowing. If the current and the last word shared a triplet in the same position (matching a potential chaining and positional representation), participants were slowed even more, as compared to when the triplet was repeated in a different position in the word (so that only the chaining representation could be effective).

In line with work separating typing into a planning phase and an execution phase (e.g., Yamaguchi \& Logan, 2014), the latency of the first keystroke was much longer than the latency of the remaining keypresses in the word. The impact of overlap of representation of serial order was mainly found for the first keystroke. This suggests that overlap of representations of serial order has an impact on the level of chunks (cf. Perlman et al., 2010). It likely affects encoding of words and planning of motor programs (cf. Yamaguchi \& Logan, 2014), while it has less influence on execution as captured in the interkeystroke intervals.

\section{EXPERIMENT 2}

We included the secondary (tone counting) task in Experiment 1 in order to avoid potential ceiling effects masking the impact of partial 
overlap of subsequently typed words with respect to the representation of serial order. In Experiment 2, we aimed at replicating the slowing effects of partial overlap between subsequently typed words and tested whether this overlap would also impact typing in a single-task block.

\section{Method}

\section{PARTICIPANTS}

Twenty participants took part in the experiment. We reported data only from 18 participants (eight females, $M_{\text {age }}=40.7 ; S D=18.4$ ). Two participants were excluded as they were unfamiliar with the German keyboard and produced high error rates in the tone counting task. All remaining participants were native speakers, had normal or corrected to normal vision, and normal hearing abilities.

\section{MATERIALS AND PROCEDURE}

Participants typed 96 words in a single-task block (typing only) and 96 words in the dual-task block. The latter was set up like the two blocks of Experiment 1: there were 24 to-be-counted tones randomly distributed across the 96 words. The order of single-task block and dual-task block were counterbalanced across participants.

\section{Results}

\section{SCREENING OF THE DATA}

The rate of words containing at least one error was $10.91 \%$ $(S D=5.25 \%)$. The mean difference between the correct number of tones and the reported number was $8.1 \%$. As in Experiment 1, there was a nonsignificant tendency for people typing faster to produce a higher proportion of words with errors, $r(18)=-.4, p=.096$. Less than one percent (i.e., $0.18 \%$ ) of the keystrokes were slower than $3 \mathrm{~s}$ and $5.14 \%$ were incorrect and excluded from analyses.

\section{WORD COMPLETION TIME}

The two-way ANOVA of word overlap (no overlap, same triplet in a different position, same triplet in the same position) and task condition (single- vs. dual-task block) only showed a main effect of word overlap, $F(1.28,21.72)=10.43, p=.002, \eta_{p}{ }^{2}=.38$. There was neither a robust main effect of task condition, $F(1,17)=3.44, p=.081$, $\eta_{\mathrm{p}}{ }^{2}=.168$, nor an interaction, $F(1.32,22.49)=2.09, p=.159$, $\eta_{\mathrm{p}}{ }^{2}=.109$. As in Experiment 1, participants were quicker to com-

\section{TABLE 2.}

Means and SDs of Response Times (ms) for Three Types of Word Overlap for Word Completion Time, Latency of First Keystroke, and Interkeystroke Interval for Experiment 1

\begin{tabular}{lccc}
\hline Measures & No overlap & $\begin{array}{c}\text { Same triplet in } \\
\text { different position }\end{array}$ & $\begin{array}{c}\text { Same triplet in } \\
\text { same position }\end{array}$ \\
\hline $\begin{array}{l}\text { Word } \\
\text { completion time }\end{array}$ & $2196(570)$ & $2278(608)$ & $2361(638)$ \\
$\begin{array}{l}\text { First keystroke } \\
\text { latency }\end{array}$ & $1045(248)$ & $1051(255)$ & $1089(308)$ \\
$\begin{array}{l}\text { Interkeystroke } \\
\text { interval }\end{array}$ & $221(71)$ & $237(77)$ & $240(72)$ \\
\hline
\end{tabular}

plete words with no overlap $(M=2039 \mathrm{~ms}, S D=542 \mathrm{~ms})$ than words with the same triplet in a different position $(M=2094 \mathrm{~ms}$, $S D=560 \mathrm{~ms}, t[17]=3.31, p=.004, d=0.78)$. Again, they were slowest when the same triplet was repeated in the same position $(M=2174 \mathrm{~ms}$, $S D=614 \mathrm{~ms}, t[17]=4.1, p=.001, d=0.97$ for the comparison with the no overlap condition, $t[17]=3.05, p=.007, d=0.72$ for the difference among the two variants of triplet overlap). Means and $S D$ s are reported in Table 3.

\section{LATENCY OF THE FIRST KEYSTROKE}

The two-factorial ANOVA again only showed a main effect of word overlap, $F(1.48,25.12)=8.52, p=.003, \eta_{\mathrm{p}}{ }^{2}=.334$ (other $\left.F \mathrm{~s}<1.1\right)$. As in Experiment 1, typing started later in words that contained the same triplet in the same position $(M=1145 \mathrm{~ms}, S D=277 \mathrm{~ms})$ than in words with no overlap $(M=1068 \mathrm{~ms}, S D=255 \mathrm{~ms}), t(17)=3.99, p=.001$, $d=0.94$. The latency in case of the overlap of the triplet in a different position $(M=1095 \mathrm{~ms}, S D=268 \mathrm{~ms})$ was higher for than no overlap, $t(17)=2.35, p=.031, d=0.55$, and lower than for the overlap of the same triplet in the same position, $t(17)=2.51, p=.023, d=0.59$.

\section{INTERKEYSTROKE INTERVAL}

Different from Experiment 1, the interkeystroke intervals were not robustly affected by word overlap, $F(2,34)=2.7, p=.081, \eta_{\mathrm{p}}{ }^{2}=.137$. However, there was a main effect of task condition, $F(1,17)=7.79$, $p=.013, \eta_{\mathrm{p}}{ }^{2}=.314$, as the interkeystroke intervals where shorter in the single-task block $(M=181 \mathrm{~ms}, S D=67 \mathrm{~ms})$ than in the dual-task block $(M=200 \mathrm{~ms}, S D=78 \mathrm{~ms})$. There was no interaction between word overlap and task condition, $F(2,34)=2.06, p=.143, \eta_{\mathrm{p}}{ }^{2}=.108$.

\section{Discussion}

Experiment 2 replicated the findings of Experiment 1 that partial overlap between subsequent words leads to costs in typing speed. There was significant additional slowing when the overlap matched the potential positional representation and the potential chaining representation rather than only the chaining representation. As in Experiment 1, we observed the overlap effects in the latencies of the first keystroke. Again, this suggests that the word-level processes rather than the execution of single keystrokes were influenced.

The impact of partial overlap between subsequently typed words did not differ between the single-task block (typing only) and the dualtask block (typing plus tone counting). Thus, ceiling effects do not seem to mask effects of overlap of representations of serial order. While our data suggest that overlap effects do occur even in single-tasking, we are hesitant to claim that dual-tasking has no effect. Future research should test whether stronger dual-task manipulations affect the impact of overlap. Testing skilled typists with a stronger dual-task manipulation might offer a good basis for this direction of research.

Obtaining overlap costs in Experiment 1 and 2 is in line with work on feature binding (e.g., Dreisbach \& Haider, 2009; Frings et al., 2007; Hommel, 1998; Hydock et al., 2013; Moeller et al., 2016; Stoet \& Hommel, 1999; Wiediger \& Fournier, 2008), which suggest that if enough stimulus features from the last trial are repeated, people might 
reuse the prior representation. Given that the typing of words seems to be executed in chunks (long response times for Letter 1, short response times for the remaining letters), activation of the representation of the prior word cannot be helpful in partial overlap. However, to support this reasoning, we need to show that full overlap between subsequent words indeed leads to a benefit in performance. In the work on feature binding (e.g., Hommel, 2004; Moeller et al., 2016), it is observed that participants were fast in case of full overlap and in case of no overlap, but slowed if there is partial overlap between the current and the last event. With partial overlap, there can be (sustained) activation of the representation from the preceding event, overcoming this activation can result in delayed processing.

Furthermore, when assessing the impact of overlap of representations of serial order on speeded typing, the impact of prior knowledge can be of interest. Yet, in Experiments 1 and 2, we used known words and could not systematically manipulate word frequency in the lexicon as we needed to pick words based on triplet position (rather than on word frequency). One way to assess the impact of prior knowledge is to contrast words and pseudo-words. Reuse of representation of serial order in speeded typing should not only speed up typing when the preceding word is (fully) repeated, but there should also be a benefit of words compared to pseudo-words.

\section{EXPERIMENT 3}

Experiment 3 explored the impact of prior knowledge by contrasting words and pseudo-words and tested whether complete overlap between subsequent (pseudo-)words can lead to a benefit in typing speed. Participants typed words and pseudo-words in separate blocks. In addition, we varied dual-tasking on the level of blocks. We enlarged the pool of material to 96 words and 96 pseudo-words. The secondary task was replaced by a tone identification task. A high and a low tone were mapped to the enter key versus the space key. Participants pressed the corresponding key after typing the word or pseudo-word.

\begin{tabular}{|c|c|c|c|}
\hline \multicolumn{4}{|c|}{$\begin{array}{l}\text { TABLE } 3 . \\
\text { Means and SDs of Response Times (ms) for Three Types of } \\
\text { Word Overlap in Single Tasking and Dual Tasking for Word } \\
\text { Completion Time, Latency of First Keystroke, and Interkey- } \\
\text { stroke Interval for Experiment } 2\end{array}$} \\
\hline Measures & No overlap & $\begin{array}{l}\text { Same triplet in } \\
\text { different position }\end{array}$ & $\begin{array}{l}\text { Same triplet in } \\
\text { same position }\end{array}$ \\
\hline \multicolumn{4}{|c|}{$\begin{array}{l}\text { Word completion } \\
\text { time }\end{array}$} \\
\hline Single & $1991(536)$ & $2053(527)$ & $2082(562)$ \\
\hline Dual & $2087(573)$ & $2145(627)$ & $2311(798)$ \\
\hline \multicolumn{4}{|c|}{$\begin{array}{l}\text { First keystroke } \\
\text { latency }\end{array}$} \\
\hline Single & $1050(240)$ & $1083(247)$ & 1097 (249) \\
\hline Dual & $1098(307)$ & $1108(323)$ & $1189(389)$ \\
\hline \multicolumn{4}{|c|}{$\begin{array}{l}\text { Interkeystroke } \\
\text { interval }\end{array}$} \\
\hline Single & $179(66)$ & $183(63)$ & $180(72)$ \\
\hline Dual & $191(70)$ & $199(81)$ & $211(85)$ \\
\hline
\end{tabular}

\section{Method}

\section{PARTICIPANTS}

Thirty German native speakers participated in Experiment 3. One participant reported hearing problems and was excluded. Fifteen participants were females $\left(M_{\text {age }}=40.3, S D=12.1\right)$.

\section{MATERIALS AND PROCEDURE}

Counterbalanced across participants, we chose different pairs of triplets (List: sch, lin, ang, ver, rei, aus, ing, and ter) for each of the different blocks. We used a Latin square (see Appendix, Table 2) to balance assignment of pairs of the above triplets to different blocks across participants.

We replaced several infrequent words from Experiments 1 and 2, for example, klinik (clinic) instead of glinka (a person's name). In total, we used a list of 96 words and 96 pseudo German words (see Appendix, Table A1). The 96 pseudo-words were created from the corresponding words. They contained the same triplets of the words, but the order of the other letters was changed such that the string was not a German word. We chose the combination that fitted the German phonology the most. For instance, one word was schaum and the corresponding pseudo-word was schmua.

There were four types of overlap in Experiment 3: no overlap (current [pseudo-]word was different from the last [pseudo-]word), same triplet in different position (current [pseudo-]word contains the same triplet but in different position as in the last [pseudo-]word), same triplet in the same position (current [pseudo-]word contains the same triplet in the same position as in the last [pseudo-]word), and full overlap (current [pseudo-]word was the same as the last [pseudo-]word).

We changed the setting for the secondary task to obtain a measure for each trial. Participants heard a tone (200 ms duration) while they typed the word. The tone was either of high $(880 \mathrm{~Hz})$ or low frequency $(440 \mathrm{~Hz})$. If the tone was high, participants needed to press the enter key to go to the next word. If the tone was low, they needed to press the space key. As soon as participant pressed the enter key or the space key, the next trial started.

Participants started with a single-task practice block containing 20 words and 20 pseudo-words (see Table 4). Then they performed one block with words and one with pseudo-words (order counterbalanced across participants). Next, they received the dual-task practice block before they performed the dual-task word typing block and the pseudo-word typing block (in counterbalanced order).

\section{Results}

\section{SCREENING OF THE DATA}

The rate of words or pseudo-words containing at least one error was $6.83 \%(S D=2.40 \%)$. The average error rate in the tone identification task was $4.71 \%(S D=3.13 \%)$. There was no indication that people typing faster would produce a higher proportion of words with errors, $r(29)=.27, p=.15$. Less than one percent (i.e., $0.003 \%$ ) of the keystrokes were slower than $3 \mathrm{~s}$ and $2.1 \%$ were incorrect and excluded 


\begin{tabular}{lcc}
\hline TABLE 4. \\
Design Overview for Experiment 3
\end{tabular}

from analyses. Typing speed for the different conditions is reported in Table 5.

\section{WORD COMPLETION TIME}

The three-way ANOVA of word overlap (no overlap, same triplet in different position, same triplet in the same position, full overlap), task condition (single- vs. dual-task block), and string type (word vs. pseudo-word) only showed a main effect of word overlap, $F(2.1,58.83)=21.69, p<.001, \eta_{\mathrm{p}}{ }^{2}=.436$, and a main effect of string type, $F(1,28)=78.65, p<.001, \eta_{\mathrm{p}}{ }^{2}=.737$. Completion time was shorter for words ( $M=1715 \mathrm{~ms}, S D=203 \mathrm{~ms}$ ) than for pseudo-words ( $M=1919 \mathrm{~ms}, S D=159 \mathrm{~ms})$. Neither the main effect of task condition, $F(1,28)=2.47, p=.127, \eta_{\mathrm{p}}^{2}=.081$, nor any interactions were significant $(F s<2.18 ; p>.10)$.

Averaged across string type, the completion time was shortest in case of full overlap $(M=1768 \mathrm{~ms}, S D=159 \mathrm{~ms})$. It differed from the no overlap condition $(M=1827 \mathrm{~ms}, S D=182 \mathrm{~ms}, t[28]=6.89$, $p<.001, d=1.28$ ), the same triplet in a different position condition $(M=1848 \mathrm{~ms}, S D=193 \mathrm{~ms}, t[28]=7.71, p<.001, d=1.43)$, and the same triplet in the same position condition $(M=1828 \mathrm{~ms}$, $S D=162 \mathrm{~ms}, t[28]=5.69, p<.001, d=1.06)$. Like in Experiments 1 and 2 , the same triplet in different position condition was significantly slower than the no overlap condition, $t(28)=3.39, p=.002, d=0.63$. This suggests that the chaining representation was used. Unexpectedly and different from Experiments 1 and 2, the same triplet in the same position condition was not slowed down. Thus, an impact of the position representation could not be documented.

\section{LATENCY OF THE FIRST KEYSTROKE}

The analogous ANOVA for latencies of the first keystroke again showed a main effect of word overlap, $F(2.24,62.82)=31.05, p<.001$, $\eta_{\mathrm{p}}^{2}=.526$. As in the above analysis, the latencies were shorter (see Table 5) for full overlap ( $M=823 \mathrm{~ms}, S D=48 \mathrm{~ms}$ ) compared to the no overlap condition $(M=864 \mathrm{~ms}, S D=57 \mathrm{~ms}, t[28]=9.07, p<.001$, $d=1.68)$, the same triplet in different position condition $(M=874 \mathrm{~ms}$, $S D=61 \mathrm{~ms}, t[28]=9.84, p<.001, d=1.83)$, and the same triplet in the same position condition $(M=858 \mathrm{~ms}, S D=58 \mathrm{~ms}, t[28]=5.21$, $p<.001, d=0.97)$. Again, the same triplet in different position condition was slower than the no overlap condition, $t(28)=2.63, p=.014$, $d=0.49$, yielding evidence for impact of the chaining representation. Yet, there was no slowing in same triplet in the same position condition (i.e., no evidence for impact of the positional representation).

Furthermore, there was a main effect of string type, $F(1,28)=114.31, p<.001, \eta_{\mathrm{p}}{ }^{2}=.803,(M=815 \mathrm{~ms}, S D=57 \mathrm{~ms}$ for words; $M=894 \mathrm{~ms}, S D=57 \mathrm{~ms}$ for pseudo-words), and a main effect of task type, $F(1,28)=19.26, p<.001, \eta_{\mathrm{p}}{ }^{2}=.408$. Single-task blocks ( $M=888 \mathrm{~ms}, S D=61 \mathrm{~ms})$ were slower than dual-task blocks $(M=822 \mathrm{~ms}, S D=54 \mathrm{~ms})$. Apparently, the impact of the secondary task was weak and a potential small effect of dual-tasking might have been masked by general practice effects (as the single-task blocks had been presented first and dual-task blocks second for all participants).

In addition to the main effects of overlap and string type - which were most relevant to the aims of the experiment-we observed three two-way interactions. The interaction of word overlap and string type, $F(1.63,45.8)=4.22, p=.008, \eta_{\mathrm{p}}{ }^{2}=.131$, was mainly driven by a larger advantage of the full overlap over the other overlap conditions in the pseudo-words as compared to the words (see Table 5). The interaction of word overlap and task condition, $F(1.74,48.8)=4.59, p=.005$, $\eta_{\mathrm{p}}{ }^{2}=.141$, was mainly due to a larger advantage of the full overlap over the other overlap conditions in the dual-task block (see Table 5). The interaction of task condition and string type, $F(1,28)=4.94, p=.035$, $\eta_{\mathrm{p}}{ }^{2}=.15$, reflected that in the dual-task blocks, the advantage of words ( $M=807 \mathrm{~ms}, S D=63 \mathrm{~ms})$ over pseudo-words $(M=871 \mathrm{~ms}$, $S D=71 \mathrm{~ms}$ ) was less pronounced than in single-task blocks ( $M=822 \mathrm{~ms}, S D=72 \mathrm{~ms}$ vs. $M=919 \mathrm{~ms}, S D=80 \mathrm{~ms}$, respectively).

\section{INTERKEYSTROKE INTERVAL}

The three-factorial ANOVA on the interkeystroke interval also showed a main effect of word overlap, $F(2.07,57)=4.32, p=.007$, $\eta_{\mathrm{p}}{ }^{2}=.134$. Full overlap again led to the fastest keystrokes ( $M=189 \mathrm{~ms}, S D=25 \mathrm{~ms}$ ) compared to the no overlap condition $(M=192 \mathrm{~ms}, S D=28 \mathrm{~ms}, t[28]=2.73, p=.011, d=0.51)$, the same triplet in a different position condition ( $M=195 \mathrm{~ms}, S D=28 \mathrm{~ms}, t[28]=3.93$, $p=.001, d=0.73)$, and the same triplet in the same position condition $(M=194 \mathrm{~ms}, S D=24 \mathrm{~ms}, t[28]=2.36, p=.026, d=0.44$, see Table 5). There was neither a slowing of the same triplet in a different position compared to no overlap $(t[28]=1.98, p=.057, d=0.37)$, nor of the same triplet in the same position keystrokes $(t[28]=0.58, p=.568)$ compared to no overlap. Furthermore, there was a main effect of string type, $F(1,28)=51.54, p<.001, \eta_{\mathrm{p}}{ }^{2}=.648(M=180 \mathrm{~ms}, S D=31 \mathrm{~ms}$ for words; $M=205 \mathrm{~ms}, S D=23 \mathrm{~ms}$ for pseudo-words; all other $F_{\mathrm{s}}<1.1$ ).

Taken together, the results suggest that full overlap (repeating the same word or pseudo-word) indeed led to a benefit in typing speed compared to no- and partial overlap at the planning (latency of first keystroke) and execution (interkeystroke intervals) level. Partial overlap of chaining representations seemed to slow down planning.

\section{Discussion}

Experiment 3 revealed that full overlap of subsequently typed words indeed leads to a benefit in speeded continuous typing. This benefit was present on the level of planning (latency of first keystroke) as well 


\section{TABLE 5.}

Means and SDs of Response Times (ms) for Four Types of Word Overlap and Two String Types (Word and Pseudo-word) in Single Tasking and Dual Tasking for Word Completion Time, Latency of First Keystroke, and Interkeystroke Interval for Experiment 3

\begin{tabular}{lcccc}
\hline Measures & No overlap & $\begin{array}{l}\text { Same triplet in } \\
\text { different position }\end{array}$ & $\begin{array}{l}\text { Same triplet in } \\
\text { the same position }\end{array}$ & Full overlap \\
\hline $\begin{array}{l}\text { Word completion time } \\
\text { Word single }\end{array}$ & $1723(287)$ & $1747(298)$ & $1739(291)$ & $1710(257)$ \\
$\quad$ Word dual & $1716(147)$ & $1740(167)$ & $1695(210)$ & $1658(172)$ \\
Pseudo single & $1960(226)$ & $1965(221)$ & $1985(251)$ & $1903(159)$ \\
Pseudo dual & $1910(188)$ & $1942(203)$ & $1904(230)$ & $1803(144)$ \\
\hline First keystroke latency & & & & \\
Word single & $827(62)$ & $832(82)$ & $823(87)$ & $804(58)$ \\
Word dual & $822(51)$ & $840(68)$ & $790(68)$ & $778(65)$ \\
Pseudo single & $926(91)$ & $926(73)$ & $938(94)$ & $888(61)$ \\
Pseudo dual & $882(71)$ & $895(67)$ & $884(94)$ & $822(54)$ \\
\hline Interkeystroke interval & & & & $184(43)$ \\
Word single & $179(47)$ & $183(46)$ & $180(36)$ & $175(25)$ \\
$\quad$ Word dual & $178(23)$ & $179(24)$ & $208(43)$ & $203(27)$ \\
$\quad$ Pseudo single & $207(33)$ & $208(35)$ & $203(34)$ & $196(23)$ \\
\hline Pseudo dual & $206(26)$ & $209(30)$ & &
\end{tabular}

as in execution (interkeystroke intervals). It was found for words as well as for pseudo-words. While the former were typed considerably faster than the latter (cf. Wilbert \& Haider, 2012), both were affected by overlap. As in Experiments 1 and 2, we found a partial overlap effect in line with the relevance of a chaining representation. Typing onset was slowed when the current (pseudo-)word shared a part of the preceding (pseudo-)word. Unexpectedly (and different from Experiments 1 and 2), there was no extra slowing when the shared part occupied the exact same position as in the preceding word. Presumably, (a) including full overlap, (b) mixing words and pseudo-words in one experiment, and/or (c) changing the secondary task to a delayed two-choice task (recognizing high or low frequency tones instead of a counting task involving updating of working memory) might have obscured the effect of partial overlap observed in Experiments 1 and 2.

It might be possible that sch or lin (used in Experiments 1 and 2) are processed as single units, similar to what has been shown for the or and in English (see, e.g., Drewnowski \& Healy, 1977; Healy, 1976 for encoding; Logan, 1982 for typing). Based on high frequency in the language (and high frequency in the material of the experiment), the letters of the triplets might be processed more quickly than other letters. In Experiment 3, we varied triplets across blocks. It is conceivable that the variation within the experiment or specific properties of the overlapping material in the language (cf. Hasenäcker, Beyersmann, \& Schroeder, 2016) might influence which effect the overlap of representation of serial order has on processing times. Future experiments might test why and how this adding of variants modulates partial overlap effects.

\section{GENERAL DISCUSSION}

This study tested whether and how two forms of representation of serial order (i.e., chaining and positional coding) impact performance in speeded typing. By varying the position of triplets overlapping between subsequent letter words, we could assess spillover effects of the activation of representation of serial order used in word typing from one word to the next. Thus, while participants in the Snyder and Logan (2014) study were confronted with a setup where primes and to-betyped words were presented, we could use a simpler setup in which participants continuously typed six-letter-words.

In all three experiments, we observed that participants were slowed down when a triplet of letters was repeated in the subsequent word at some other positions. Thus, while the priming procedure in the Snyder and Logan (2014) study led to an increase in typing speed, we observed costs when the current word partially overlapped with the previous one in speeded continuous typing. Partitioning overall word completion time into latency of the first keystroke (as a measure of planning, see Yamaguchi \& Logan, 2014) and interkeystroke intervals (i.e., execution), we found evidence that the overlap effects were most pronounced in encoding of words and planning of motor programs. Presumably, partial overlap led to sustaining activation of the representation of the previous word which impeded the activation of the motor programs of the currently to-be-typed word.

Slow latencies on the first letter paired with fast keypresses for the remaining letters are consistent with the view that the letters were activated before the first key was pressed and were executed as a chunk (e.g., Perlman et al., 2010). In line with the view that overlap effects operate on the word level, our study showed that effects of partial overlap were mainly driven by the latency of the first keypress rather than by the interkeystroke intervals. Whole-word-effects make it plausible that the overlap between the preceding and the current word resulted in continued activation of the previous word which might have interfered with the current word. Costs of partial overlap (Experiments 1 and 2) and a benefit in case of full overlap (Experiment 3 ) have been observed in studies on feature binding (e.g. Hommel, 1998; Wiediger \& Fournier, 2008). By using a sign when a word was finished and by pre- 
senting single words on the screen, we likely provided strong cues with respect to when updating of representation of serial order was needed. This might have contributed to finding word level effects. Note, however, that the literature on chunking has documented that cues are used in sequence learning to separate different chunks as well (cf. Hoffman et al., 2017; Perlman, Hoffman, Tzelgov, Pothos, \& Edwards, 2016; Perlman et al., 2010). If practiced sequences differ in frequency and are presented with cues that allow to separate them into different chunks, even identical transitions of keystrokes are executed at different speed, suggesting that, to a large extent, sequence knowledge is used at the level of chunks rather than at the level of single transitions of keystrokes or position of keystrokes in a sequence. These studies show that unitization effects are not limited to material where one potential unit in a series of keystrokes ends and the next one begins. Rather, chunk level representations seem to be generated even when chunk borders have to be extracted from cues.

In Experiments 1 and 2, word completion times and latency of first keystroke suggested that partial overlap of subsequent words led to the largest slowing when the overlapping triplet occurred in the same position in the word (but see the Discussion section of Experiment 3). Probably, the representation of serial order of the letters in the words contained positional information in addition to chaining information. While Snyder and Logan (2014) argued exclusively for chaining, our findings point towards the involvement of both forms of representation of serial order in speeded continuous typing. This is in line with studies on implicit sequence learning suggesting that both forms can be acquired and operate in parallel (cf. Schuck, Gaschler, \& Frensch, 2012).

The current study involves tradeoffs in the choice of methods that should be explored systematically in future work. We used continuous typing of random word sequences and coded overlap between subsequent words as they occurred in random sequences. This could avoid leading participants to expect certain variants of overlap to occur. We know little about the effects of partial overlap in subsequent words in natural language, apart from the fact that some variants of poems are characterized by high overlap in subsequent words (cf. Dhami, Hertwig, \& Hoffrage, 2004, for a discussion of representative design as an ecological approach to cognition). All words in this study were randomly distributed, thus, the word overlap conditions appeared in different frequencies. Based on the number of words, no overlap was more common than partial overlap of elements in different positions in the word. Partial overlap in the same position in the word was least common. By systematically changing the size of the word pool, the frequency of these cases of overlap could be varied systematically. Once it is known whether and how participants adapt to changes in frequency of overlap variants, it would be possible to gauge the advantages of systematically pairing words as predecessors and successors, such that the role of words is fully balanced. The number of cases of measurement could then be optimized to gain the most stable estimates of typing speed per type of overlap possible. Apart from this, future studies might test the potential impact of ceiling effects with more difficult secondary tasks. Our manipulation of task condition was mostly weak (i.e., no consistent main effect in Experiments 2 and 3, where single-tasking blocks could be compared with dual tasking blocks), suggesting that our dual-task manipulation would not have been effective enough in increasing task difficulty to prevent potential ceiling effects.

The setup used in the current study might lay the ground for studying short-term (i.e., the overlap between subsequent words) and long-term (i.e., sequence knowledge based on multiple blocks of practice) effects of usage of and change in representations of serial order (cf. Yamaguchi \& Logan, 2016, for impact of memory load on storing of typed words and nonwords as chunks in long-term memory). Dynamics in representation of serial order have, so far, either been studied from trial to trial or across blocks and sessions of practice. For instance, Schuck et al. (2012) showed that the impact of ordinal position coding on behaviour might outweigh the impact of chaining early in practice, but chaining might become dominant across sequence learning sessions. On the other hand, modelling suggests that it should be feasible to treat short-term and long-term dynamics in representation of serial order in a common framework. Botvinick and Plaut (2006) used a recurrent network model (similar to models of implicit sequence learning, e.g., Cleeremans \& McClelland, 1991; Elman, 1990; Pacton, Perruchet, Fayol, \& Cleeremans, 2001) to account for (short-term) benchmark effects of chaining and ordinal position knowledge within one artificial neuronal network framework. Paralleling the convergence in modelling long-term and short-term effects in representation of serial order, training of speeded typing of (pseudo-)words with partial overlap in subsequent items might be used to capture short-term and long-term dynamics in representations of serial order in one experimental paradigm.

\section{FOOTNOTES}

${ }^{1}$ To account for the within-subjects design, we computed $d$ by dividing the mean difference score by the $S D$ of the difference scores (see $d_{z}$, Cohen, 1988, p. 48).

${ }^{2}$ In order to further disentangle potential planning and execution effects, we checked whether the effect of triplet overlap occurred irrespective of the position of the triplet in the word or differed when the triplet involved the start of the word. The former seemed to be the case. A two-factorial ANOVA involving overlap (see above) and triplet (typed letter belongs to a triplet vs. does not belong to a triplet) again documented the main effect of overlap, $F(1.46,36.47)=4.19, p=.034$, $\eta_{\mathrm{p}}{ }^{2}=.143$. There was a main effect of triplet, $F(1,25)=84.5, p<.001$, $\eta_{\mathrm{p}}{ }^{2}=.772$, as triplet letters $(M=920 \mathrm{~ms}, S D=268 \mathrm{~ms})$ were typed faster than other letters $(M=1084 \mathrm{~ms}, S D=273 \mathrm{~ms})$. There was no interaction $(F<1)$.

${ }^{3}$ A separate analysis of keystrokes from triplets and other letters was run to explore whether slowing occurs due to interference with retrieval or execution of the overlapping letters themselves or of the entire word in the interkeystroke intervals. The two-factorial ANOVA involving overlap and triplet (typed letter belongs to a triplet vs. does not belong to a triplet) again documented the main effect of overlap, $F(2,50)=12.85, p<.001, \eta_{\mathrm{p}}{ }^{2}=.339$, and of triplet, $F(1,25)=37.46, p<$ $.001, \eta_{\mathrm{p}}{ }^{2}=.6$. The interaction, $F(1.24,31.2)=9.88, p=.002, \eta_{\mathrm{p}}{ }^{2}=.283$, was driven by a large difference $(M=59 \mathrm{~ms})$ between triplet- versus 
nontriplet letters in case of overlap of triplet and position, while this difference was smaller in case of no overlap $(M=29 \mathrm{~ms})$ and same triplet in different positions $(M=25 \mathrm{~ms})$. Given that the interkeystroke interval differed more for nontriplet letters $(M=236 \mathrm{~ms} ; M=251 \mathrm{~ms}$, and $M=273 \mathrm{~ms}$, for no-, triplet-, and position overlap, respectively) than for triplet letters ( $M=208 \mathrm{~ms} ; M=226 \mathrm{~ms}, M=213 \mathrm{~ms})$, the results seem to suggest that interkeystroke intervals were not delayed by the execution of the overlapping letters themselves. Note that we are hesitant to fully interpret these findings as the interaction was not replicated in Experiment 2.

\section{ACKNOWLEDGEMENTS}

This research was supported by grants within the Priority Program, SPP 1772 from the German Research Foundation (Deutsche Forschungsgemeinschaft, DFG), grants no [GA 2246/1-1; HA 5447/111]. We thank FernUniversität in Hagen for support of this work and its publication.

\section{REFERENCES}

Botvinick, M. M., \& Plaut, D. C. (2006). Short-term memory for serial order: A recurrent neural network model. Psychological Review, 113, 201-233. doi: 10.1037/0033-295X.113.2.201 |wWW

Cleeremans, A., \& McClelland, J. L. (1991). Learning the structure of event sequences. Journal of Experimental Psychology. General, 120, 235-253. doi: 10.1037//0096-3445.120.3.235 WWW

Cohen, J. (1988). Statistical power analysis for the behavioral sciences. Hillsdale, NJ: Lawrence Earlaum Associates.

Dhami, M. K., Hertwig, R., \& Hoffrage, U. (2004). The role of representative design in an ecological approach to cognition. Psychological Bulletin, 130, 959-988. doi: 10.1037/00332909.130.6.959 WWW

Dreisbach, G., \& Haider, H. (2009). How task representations guide attention: further evidence for the shielding function of task sets. Journal of Experimental Psychology. Learning, Memory, and Cognition, 35, 477-486. doi: 10.1037/a0014647 WWW

Drewnowski, A., \& Healy, A. F. (1977). Detection errors on the and and: Evidence for reading units larger than the word. Memory \& Cognition, 5, 636-647. doi: 10.3758/BF03197410 |wWW

Ebbinghaus, H. (1885). Über das Gedächtnis [Memory: A contribution to experiemental psychology]. (H. A. Ruger \& C. E. Bussenius, Trans.). Darmstadt, Germany: Wissenschaftliche Buchgesellschaft.

Elman, G. (1990). Finding structure in time. Cognitive Science, 14, 179-211. doi: $10.1207 / \mathrm{s} 15516709 \operatorname{cog} 1402 \_1$

Frings, C., Rothermund, K., \& Wentura, D. (2007). Distractor repetitions retrieve previous responses to targets. Quarterly Journal of Experimental Psychology, 60, 1367-1377. doi: 10.1080/17470210600955645 WWW

Hasenäcker, J., Beyersmann, E., \& Schroeder, S. (2016). Masked morphological priming in German-speaking adults and children: Evidence from response time distributions. Frontiers in Psychology, 7:929, 1-11. doi: 10.3389/fpsyg.2016.00929 www
Healy, A. F. (1976). Detection errors on the word the: Evidence for reading units larger than letters. Journal of Experimental Psychology: Human Perception and Performance, 2, 235-242. doi: 10.1037/0096-1523.2.2.235 WwW

Henson, R. N. A. (1998). Short term memory for serial order: The start-end model. Cognitive Psychology, 28, 527-545. doi: 10.1006/cogp.1998.0685 WwW

Hoffman, Y., Perlman, A., Orr-Urtreger, B., Tzelgov, J., Pothos, E. M., \& Edwards, D. J. (2017). Unitization of route knowledge. Psychological Research, 81, 1241-1254. doi: 10.1007/s00426016-0811-0 WWW

Hommel, B. (1998). Event files: Evidence for automatic integration of stimulus-response episodes. Visual Cognition, 5, 183-216. doi: $10.1080 / 713756773$

Hommel, B. (2004). Event files: Feature binding in and across perception and action. Trends in Cognitive Sciences, 8, 494-500. doi: 10.1016/j.tics.2004.08.007 |WWW

Houghton, G., \& Hartley, T. (1996). Parallel models of serial behaviour: Lashley revisited. Psyche: An Interdisciplinary Journal of Research on Consciousness, 2, 1-25.

Hydock, C., Patai, E. Z., \& Sohn, M. H. (2013). Distinct response components indicate that binding is the primary cause of response repetition effects. Journal of Experimental Psychology. Human Perception and Performance, 39, 1598-1611. doi:

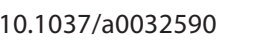

Kezilas, Y., McKague, M., Kohnen, S., Badcock, N., \& Castles, A. (2017). Disentangling the developmental trajectories of letter position and letter identity coding using masked priming. Journal of Experimental Psychology. Learning, Memory, and Cognition, 43, 250-258. doi: 10.1037/xIm0000293 |WWW

Lashley, J. K. (1951). The problem of serial order in behavior. In L. A. Jeffress (Ed.), Cerebral mechanisms in behavior. (pp. 112-136). New York, NY: Wiley.

Logan, G. D. (1982). On the ability to inhibit complex movements: A stop-signal study of typewriting. Journal of Experimental Psychology: Human Perception and Performance, 8, 778-792. doi: 10.1037/0096-1523.8.6.778

Moeller, B., Pfister, R., Kunde, W., \& Frings, C. (2016). A common mechanism behind distractor-response and response-effect binding? Attention, Perception, \& Psychophysics, 78, 1074-1086. doi: 10.3758/s13414-016-1063-1 $\underline{\underline{W W}}$

Pacton, S., Perruchet, P., Fayol, M., \& Cleeremans, A. (2001). Implicit learning out of the lab: The case of orthographic regularities. Journal of Experimental Psychology. General, 130, 401-426. doi: 10.1037/0096-3445.130.3.401 WWW

Peirce, J. W. (2007). PsychoPy—Psychophysics software in Python. Journal of Neuroscience Methods, 162, 8-13. doi: 10.1016/j. jneumeth.2006.11.017 WWW

Perlman, A., Hoffman, Y., Tzelgov, J., Pothos, E. M., \& Edwards, D. J. (2016). The notion of contextual locking: Previously learnt items are not accessible as such when appearing in a less common context. The Quarterly Journal of Experimental Psychology, 
69, 410-431. doi: 10.1080/17470218.2015.1054846 WWW

Perlman, A., Pothos, E. M., Edwards, D. J., \& Tzelgov, J. (2010). Task-relevant chunking in sequence learning. Journal of Experimental Psychology. Human Perception and Performance, 36, 649-661. doi: 10.1037/a0017178 $\underline{\underline{\mathrm{ww}}}$

Rumelhart, D. E., \& Norman, D. A. (1982). Simulating a skilled typist: A study of skilled cognitive-motor performance. Cognitive Science, 6, 1-36. doi: 10.1207/s15516709cog0601_1

Schuck, N. W., Gaschler, R., \& Frensch, P. A. (2012). Implicit learning of what comes when and where within a sequence: The timing-course of acquiring serial position-item and item-item associations to represent serial order. Advances in Cognitive

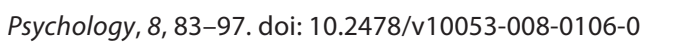

Snyder, K. M., \& Logan, G. D. (2014). The problem of serial order in skilled typing. Journal of Experimental Psychology. Human Perception and Performance, 40, 1697-1717. doi: 10.1037/ a0037199 $\overline{\text { WWW }}$

Stoet, G., \& Hommel, B. (1999). Action planning and the temporal binding of response codes. Journal of Experimental Psychology. Human Perception and Performance, 25, 1625-1640. doi: 10.1037/0096-1523.25.6.1625
Wiediger, M. D., \& Fournier, L. R. (2008). An action sequence withheld in memory can delay execution of visually guided actions: The generalization of response compatibility interference. Journal of Experimental Psychology: Human Perception and Performance, 34, 1136-1149. doi: 10.1037/00961523.34.5.1136 $\overline{\mathrm{WWW}}$

Wilbert, J., \& Haider, H. (2012). The subjective experience of committed errors and the Discrepancy-Attribution hypothesis. Acta Psychologica, 139, 370-381. doi: 10.1016/j.actpsy.2011.11.010 WWW

Yamaguchi, M., \& Logan, G. D. (2014). Pushing typists back on the learning curve: revealing chunking in skilled typewriting. Journal of Experimental Psychology. Human Perception and Performance, 40, 592-612. doi: 10.1037/a0033809 |wWw

Yamaguchi, M., \& Logan, G. D. (2016). Pushing typists back on the learning curve: Memory chunking in the hierarchical control of skilled typewriting. Journal of Experimental Psychology: Learning, Memory, and Cognition, 42, 1919-1936. doi: 10.1037/ $x \operatorname{lm} 0000288 \underline{\underline{W W}}$

RECEIVED 01.09.2017| ACCEPTED 29.06.2018 


\section{APPENDIX}

TABLE A1.

All Words and Pseudo-Words Used in Experiment 3

\begin{tabular}{|c|c|c|c|c|c|c|}
\hline Triplet & Position & Word & Pseudo-word & Position & Word & Pseudo-word \\
\hline Triplet pair & rat*** & ration & ratoni & ein*** & einzug & einguz \\
\hline \multirow[t]{11}{*}{ "rat" + "ein" } & rat*** & ratsam & ratmas & ein*** & einsam & einams \\
\hline & rat*** & ratten & ratent & ein*** & einige & eingei \\
\hline & *rat** & krater & eratkr & $*$ ein $* *$ & beinah & heinba \\
\hline & *rat** & fratze & zratef & *ein** & keiner & reinke \\
\hline & *rat** & gratis & sratgi & *ein** & feinde & deinef \\
\hline & **rat* & karate & ekrata & **ein* & boeing & obeing \\
\hline & **rat* & sorata & asrato & **ein* & steine & eseint \\
\hline & **rat* & herati & ihrate & **ein* & uneins & sneinu \\
\hline & ***rat & heirat & iherat & $\star * \star$ ein & allein & lalein \\
\hline & ***rat & vorrat & orvrat & $\star * \star$ ein & gemein & emgein \\
\hline & ***rat & baurat & aubrat & ${ }^{\star * \star}$ ein & schein & chsein \\
\hline Triplet pair & $\operatorname{sch}^{* \star \star}$ & schaum & schmua & lin*** & lineal & linlae \\
\hline \multirow[t]{11}{*}{ "sch" + "lin" } & $\operatorname{sch} * \star \star$ & scherz & schrez & $\operatorname{lin} * * *$ & linken & linnek \\
\hline & $\operatorname{sch}^{\star \star *}$ & schote & schteo & $\operatorname{lin} * * *$ & lindau & linuda \\
\hline & $* \operatorname{sch} * *$ & ascher & eschra & *lin** & flinte & tlinef \\
\hline & $* \operatorname{sch} * *$ & ischia & aschii & *lin** & clinch & hlince \\
\hline & ${ }^{*} \operatorname{sch} * *$ & tschad & aschdt & *lin** & klinik & klinki \\
\hline & $* * \operatorname{sch} *$ & dusche & edschu & ** $\operatorname{lin} *$ & splint & tslinp \\
\hline & ${ }^{* \star} \operatorname{sch}{ }^{*}$ & gischt & itschg & $\star \star \operatorname{lin} *$ & reling & eglinr \\
\hline & $* \star \operatorname{sch} *$ & pascha & apscha & $* \star \operatorname{lin} *$ & saline & eslina \\
\hline & $\star * \star \operatorname{sch}$ & barsch & arbsch & $\star * \star \operatorname{lin}$ & berlin & erblin \\
\hline & $\star \star \star \star s c h$ & mensch & nemsch & $\star \star \star \star \operatorname{lin}$ & dublin & ubdlin \\
\hline & $\star \star \star \star s c h$ & frosch & rofsch & $\star * \star \operatorname{lin}$ & myelin & yemlin \\
\hline Triplet pair & ang*** & angler & angrel & ver*** & verbot & vertob \\
\hline \multirow[t]{11}{*}{ "ang" + "ver" } & ang*** & angola & anglao & ver*** & verlag & vergla \\
\hline & $\operatorname{ang} * \star \star$ & angabe & angeab & ver*** & verein & verine \\
\hline & *ang** & fangen & nangfe & *ver** & averna & averan \\
\hline & $\star$ ang ${ }^{* \star}$ & mangos & sangmo & *ver** & iverni & iverin \\
\hline & *ang** & hangar & rangha & *ver** & everts & sveret \\
\hline & **ang* & stange & esangt & ** ver* & divers & isverd \\
\hline & **ang* & orange & reango & **ver* & adverb & baverd \\
\hline & **ang* & django & joangd & **ver* & kuvert & utverk \\
\hline & $\star * \star$ ang & anfang & fnaang & $\star * \star$ ver & clever & lecver \\
\hline & $\star \star \star \star a n g$ & gesang & esgang & $\star \star \star \star$ ver & pulver & lupver \\
\hline & ***ang & zugang & guzang & ***ver & oliver & ilover \\
\hline Triplet pair & rei*** & reifen & reinef & aus*** & auster & ausert \\
\hline \multirow[t]{11}{*}{ "rei" + "aus" } & rei*** & reihum & reimuh & aus*** & ausbau & ausabu \\
\hline & $r e i * \star \star$ & reisig & reigis & aus*** & ausweg & ausgew \\
\hline & *rei** & breite & treieb & *aus** & rausch & caushr \\
\hline & *rei** & dreist & sreidt & *aus** & kausal & lauska \\
\hline & *rei** & freier & ereifr & *aus** & pausen & nauspe \\
\hline & **rei* & streik & ksreit & **aus* & brause & reausb \\
\hline & **rei* & anreiz & zarein & **aus* & krauss & srausk \\
\hline & **rei* & bereit & etreib & **aus* & flause & efausl \\
\hline & $\star * \star$ rei & schrei & chsrei & $\star \star \star \star a u s$ & daraus & radaus \\
\hline & ***rei & unfrei & nufrei & ***aus & zuhaus & huzaus \\
\hline & $\star \star \star \star r e i$ & tuerei & eutrei & $\star \star \star \star a u s$ & hinaus & nihaus \\
\hline Triplet pair & ing*** & ingwer & ingrew & ter*** & termin & ternim \\
\hline \multirow[t]{11}{*}{ "ing" + "ter" } & ing*** & ingrid & ingdir & $\operatorname{ter} * \star \star$ & terror & terorr \\
\hline & ing*** & ingoda & ingaod & $\operatorname{ter} * * *$ & terzel & terlze \\
\hline & *ing** & ringen & ningre & $\star$ ter ${ }^{* *}$ & sterne & eterns \\
\hline & *ing** & finger & ringfe & $\star$ ter $* *$ & steril & iterls \\
\hline & *ing** & single & linges & $*$ ter** & uterus & utersu \\
\hline & **ing* & klinge & elingk & $* * \operatorname{ter} *$ & altert & taterl \\
\hline & **ing* & twingo & owingt & **ter* & ostern & noters \\
\hline & **ing* & frings & rsingf & **ter* & intern & nitern \\
\hline & $\star \star \star \star$ ing & unding & duning & $\star * \star \star t e r$ & krater & rakter \\
\hline & $\star \star \star \star$ ing & hering & rehing & $\star \star \star \star \operatorname{ter}$ & hefter & fehter \\
\hline & $\star \star \star$ ing & doping & opding & ***ter & gitter & tigter \\
\hline
\end{tabular}




\section{TABLE A2.}

All Participants were Counterbalanced in a Latin Square to Avoid the Sequence Effect of the Triplets. Sch + Lin in Block 1 Represented that All the Words and Pseudo-Words in This Block Contain Either the Triplet Sch or The Triplet Lin

\begin{tabular}{ccccc}
\hline Participants & Block 1 & Block 2 & Block 3 & Block 4 \\
\hline $01-08$ & sch + lin & ang + ver & rei + aus & ing + ter \\
$09-16$ & ang + ver & sch + lin & ing + ter & rei + aus \\
$17-24$ & rei + aus & ing + ter & sch + lin & ang + ver \\
$25-32$ & ing + ter & rei + aus & ang + ver & sch + lin \\
\hline
\end{tabular}

